\title{
Study on MRI and CT Combined Diagnosis Technology Application in Brain Glioma Diagnosis
}

\author{
Shi Hong-yan, Wu Xiao-qiang*, Wang Li-hua, Li Li
}

Inner Mongolia University for the Nationalities, College of Mechanical Engineering, Tongliao 028000, China

\begin{abstract}
Objective: To study the application of CT and MRI fusion technique in the diagnosis of brain glioma. Methods: 20 cases of brain glioma patients in the Affiliated Hospital of Inner Mongolia University for the Nationalities during Jan., 2014 to Dec., 2014 were selected as the study group. The study group patients accepted CT and MRI scan, and then through the fusion technology the MRI images were mapped to CT images. the rolling shutter technology was used in the CT images to determine the human body contour and other hard tissue such as bones, and MRI images were used to determine the target area boundary and other soft tissue. While another 6 cases of brain glioma patients who only accepted CT scan were selected as control group. Results: The study showed that CT and MRI fusion technology has greatly improved the outline of the target area, the normal organization, the reliability of the outline of the dangerous organs in study group. The reliability and accuracy of the clinical diagnosis of the study group were significantly higher than that of the control group( $(\mathrm{P}<0.05)$. Conclusion: The MRI and CT combined diagnosis technology can improve the clinical diagnosis of cerebral gliomas greatly.
\end{abstract}

Keywords: MRI, CT, Brain Glioma

\section{Introduction}

CT image is always the best image resource in 3DCRT target area and the risk of organ delineation, because CT has poor distinguish ability to the soft tissue structure, which has the similar electron density, when it carries on target delineation for the patients who suffered the surgery of radiotherapy for intracranial tumor, due to edema and the presence of normal anatomy, as well as the changes in CT after surgery, it is difficult to define the CTV, GTV, which is easy to cause the target delineation uncertainty, thus it can affect the efficacy of radiation therapy[1]. MRI has a high resolution of soft tissue, but it can not be directly used for radiotherapy treatment planning. If the two images can be fused together, the accuracy of target delineation can be improved by combining the advantages of the two images. Then we can combine the advantages of CT and MRI image fusion together so as to improve its accuracy[2]. In this paper, we used 20 cases of brain glioma in our hospital since 2005, positioning with mask fixation and mask in vitro marker, so as to discuss the changes of CTV and GTV after fusion.

\section{Object and Method}

\section{Preparing and Making Vitro Labeling of CT and MRI}

The mixture of lohexol and Gd-DTPA( $1.61 \mathrm{mg} / \mathrm{ml})$ was diluted to form a kind of 6:1 
mixture, which diameter is about $2 \mathrm{~mm}$, and the three spheres can be fixed on the mold as well as the mask.

\section{Clinical Data}

From January to December in 2014, 20 patients with glioma were confirmed by surgery and pathology in Affiliated Hospital of Inner Mongolia University. There were a total of 15 cases of males and 5 cases of females, aged from 16 years old to 69 years old, and the average age was 43.6 years old. Among 20 patients, 15 cases of patients had headache and dizziness in 5 cases of patients had seizures. All patients showed glioma with preoperative MRI and CT examination[3]. After having operation, 8 cases of patients had astrocytoma from grade 2 to grade 3, and there were 2 cases of glioblastoma in the brain, 9 cases of glioblastoma, 1 case of glioma. All patients underwent 3D-CRT after operating with one month. The delineation of the target area of the postoperative radiotherapy of glioma was sketched on the CT image, and the dose was calculated and verified. On the basis of CT positioning combined with MRI, the radiotherapy target area was modified again. All patients were treated with radiotherapy, after that it must have the oral consent from the patients themselves or and their family members to carry on MRI.

\section{Method and Statistical Processing}

There are 20 cases of patients with 3DCRT, applying Marconi simulation of multi-slice spiral CT and 1.5TMarconi superconducting MRI instrument, MRI positioning can use soft package phased array coil, 3DCRT planning system by Daheng Star2000 treatment planning system. After fixing the mask, the previous CT can have simulation positioning, scanning parameters: $120 \mathrm{kv}, 220 \mathrm{mAs}$, which layer thickness is $5.0 \mathrm{~mm}$, FOV240mm. The scanning range can be from head skin to clavicle. Enhanced injection of lohexol from $80 \mathrm{ml}$ hand vein, the flow rate is $23 \mathrm{ml} / \mathrm{s}$. It can scan after 85 s later. After CT scanning in MR immediately after transection T1WI (TR300ms TE20ms) and T2WI (TR3500msTE120ms), FOV, scanning direction, angle, thickness and range can be simulated with CT, CT and MR, image registration is the standard of having successful image match between $\mathrm{CT}$ and MR is that there is the same image positioning on these four spheres both on CT image and MR image, which are on display the same level. Three doctors draw GTV on the CT and MR for these twenty patients with SPSS14.0 software so as to have statistical analysis. Then they can take CT-GTV as the independent variable, MRI/T-GTV as the dependent variable, so as to carry on the linear regression to calculate the regression coefficient, and the normal distribution can be paired by using $t$ test.

\section{Result}

The location image of 20 cases of the preparation of lohexol and Gd-DTPA diluted mixture solution, with the volume ratio 6:1 can be clearly presented, the sphere can have high density and no artifact on the CT image, which showed high signal on each sequence of MRI, and the image was not deformed. GTV and CTV changes of 20 patients with CT and MRI delineation in target area can be shown in Table 1. 
Table 1 Changes of GTV and CTV of 20 Patients' CT and MRI in the Target Area

\begin{tabular}{|c|c|c|c|c|}
\hline \multirow{2}{*}{$\frac{\text { Number }}{1}$} & \multicolumn{2}{|c|}{$\mathrm{CT}$} & \multicolumn{2}{|c|}{ MRI } \\
\hline & 286.75 & 426.35 & 345.69 & 471.58 \\
\hline 2 & 350.21 & 463.43 & 210.32 & 331.54 \\
\hline 3 & 183.28 & 267.04 & 288.51 & 299.12 \\
\hline 4 & 141.54 & 358.43 & 447.64 & 532.32 \\
\hline 5 & 199.67 & 218.83 & 320.44 & 379.64 \\
\hline 6 & 278.31 & 410.79 & 335.15 & 451.23 \\
\hline 7 & 268.36 & 390.45 & 298.15 & 425.18 \\
\hline 8 & 198.56 & 252.52 & 147.32 & 281.24 \\
\hline 9 & 296.92 & 396.68 & 263.73 & 277.32 \\
\hline 10 & 310.68 & 481.36 & 400.62 & 510.23 \\
\hline 11 & 202.18 & 334.32 & 296.12 & 358.31 \\
\hline 12 & 123.92 & 190.94 & 101.71 & 150.21 \\
\hline 13 & 108.51 & 131.61 & 121.79 & 150.73 \\
\hline 14 & 169.64 & 279.51 & 212.53 & 299.76 \\
\hline 15 & 175.15 & 348.26 & 250.43 & 384.21 \\
\hline 16 & 158.82 & 495.53 & 291.16 & 531.21 \\
\hline 17 & 79.42 & 160.42 & 168.26 & 190.16 \\
\hline 18 & 89.01 & 151.55 & 102.3 & 188.53 \\
\hline 19 & 122.92 & 170.42 & 101.61 & 160.54 \\
\hline 20 & 122.43 & 192.92 & 84.16 & 210.21 \\
\hline Average value & 193.31 & 306.07 & 239.38 & 329.16 \\
\hline
\end{tabular}

We can see from the comparison of different volume in target delineation area through CT and MR, among 20 cases, there are 3 patients with confirmed GTV and CTV, whose MR is less than CT, while the other patients' GTV and CTVMR were greater than CT, namely, GTV can be increased 23.8\%, CTV can be increased 7.5\%. Comparing MR and CT image fusion with CT, GTV can be increased, $t=5.393, \mathrm{P}=0.000$, as for the regression coefficient, $\mathrm{b}=0.754$, namely, MR and CT image fusion can increase GTV, which can have significance. Comparing MR and CT image fusion with CT, GTV can be increased, $t=7.236, \mathrm{P}=0.000$, as for the regression coefficient, $b=0.579$, namely, MR and CT image fusion can increase CTV, which can have significance. The range of the regression coefficient of CT, as well as CT and MR fusion among these 20 patients is between 0.579 and 0.754 , which can indicate that the volume deviation between MR/CT-GTV and CT-GTV is between 25\% to $40 \%$.

\section{Conclusion}

MRI signal is mainly a reflection of the relaxation time and proton density, which can not reflect the electron density, therefore, MRI gray can not be directly used for calculation and verification of radiation dose, it must be corrected or conversed so as to be applied in 
radiotherapy planning system, which can limit the application in radiotherapy planning system. In addition, MRI is easy to deform, especially in the air and bone interface on the magnetic susceptibility artifacts caused by image distortion, the image artifact has little effect on disease diagnosis, however it can have great impact on 3DCRT. Therefore, if the preparation of CT and MRI is applied to 3DCRT, it can develop its advantages and avoid its weaknesses. To prepare CT and MRI, first of all it needs to prepare CT and MRI solution that can be clearly displayed in vitro labeling. This study uses the location of markers of lohexol and Gd-DTPA dilution with volume ratio 6:1, which can be clearly shown in CT and MRI[4]. Thus it can have high density on CT images, which can present high signal in the sequence of MRI and image can have no deformation. 20 cases of patients' positioning on the mask with 3 markers on CT and MRI can be at the same level, the registration effect of CT and MRI is very good. In addition, the anatomical structure of the brain in the CT and MR can be at the same level, which can indirectly determine whether the integration of CT and MRI success or not. The gray level of CT image can reflect the electron density, the dose distribution in vitro can be related to the electron density of the tissue. Moreover, CT image is smaller than MRI image, so CT image has been used as the radiotherapy plan. But CT's soft tissue resolution is low, which can not accurately draw the delineation of normal tissue morphology, such as brain gray matter, as for radiotherapy planning system, CT image target delineation is mainly adopted, because after the operation, the changes of CT is difficult to define CTV as well as the surrounding normal brain tissue, which can affect the accuracy of brain radiotherapy. Although the MRI image can have better identification for the soft tissue structures, but due to the electron density image, deformation as well as the lack of the required dose limits in the treatment planning in radiation, thus it can be an important way to combine the them, so as to improve the accuracy of radiotherapy effect.

\section{Acknowledgement}

This work is supported by The Inner Mongolia Autonomous Region Natural Science Foundation: 2016MS0550

\section{Reference}

[1] Swanson K R, Bridge C, Murray J D, et al. Virtual and real brain tumors: using mathematical modeling to quantify glioma growth and invasion[J]. Journal of the neurological sciences, 2003, 216(1): 1-10.

[2] Kitagawa Y, Nishizawa S, Sano K, et al. Prospective comparison of 18F-FDG PET with conventional imaging modalities (MRI, CT, and 67Ga scintigraphy) in assessment of combined intraarterial chemotherapy and radiotherapy for head and neck carcinoma[J]. Journal of Nuclear Medicine, 2003, 44(2): 198-206.

[3] Boss A, Bisdas S, Kolb A, et al. Hybrid PET/MRI of intracranial masses: initial experiences and comparison to PET/CT[J]. Journal of Nuclear Medicine, 2010, 51(8): 1198-1205.

[4] Burger P C, Heinz E R, Shibata T, et al. Topographic anatomy and CT correlations in the untreated glioblastoma multiforme[J]. Journal of neurosurgery, 1988, 68(5): 698-704. 